\title{
ONE-SIDED MINIMAL SURFACES WITH A GIVEN BOUNDARY*
}

\author{
BY \\ JESSE DOUGLAS

\section{HistoricAL}

One-sided surfaces present themselves quite naturally in the theory of minimal surfaces; they were first studied systematically by Sophus Lie $\dagger$ under the name of "Minimaldoppelflächen."

Lie had interpreted the formulas of Monge for a minimal surface in the now well known geometric way: that every minimal surface can be generated by translating one minimal curve $\ddagger$ along another. An equivalent construction is to take the mid-points of all line segments whose ends lie respectively on any two fixed minimal curves $\mu_{1}, \mu_{2}$; their locus is a minimal surface, which is real whenever $\mu_{1}, \mu_{2}$ are conjugate complex. If $\mu_{1}$ and $\mu_{2}$ coincide in a minimal curve $\mu$, this construction becomes the taking of the locus of the midpoints of all chords of $\mu$. The resulting minimal surface, real whenever $\mu$ is its own conjugate complex curve, is of the type designated as "Minimaldoppelfläche" by Lie. If this surface is not periodic, i.e. does not go over into itself by a certain translation and its repetitions, then it is a one-sided surface in the sense of topology: a,material point moving continuously on the surface can pass from any position to that directly beneath without crossing over any boundary of the surface (Möbius strip); or, if we ascribe a certain arrow to the normal at any fixed point and follow the continuous variation of this sensed normal as the point moves on the surface, it is possible to describe a closed path which will reverse this arrow. In particular, the surface cannot be periodic if it is algebraic; therefore,every algebraic double minimal surface is one-sided. Algebraic character can be secured for the surface by taking the minimal curve $\mu$ to be algebraic; this in turn can be done by taking as algebraic the arbitrary function $f(t)$ in the Weierstrass formulas for a minimal curve:§

* Preliminary communication presented to the Society, October 25, 1930; Bulletin of the American Mathematical Society, vol. 36 (1930), p. 797. Received by the editors June 3, 1932.

$\dagger$ S. Lie, Beiträge sur Theorie der Minimalfä̈chen, Mathematische Annalen, vol. 14 (1878), p. 331 ; vol. 15 (1879), p. 465.

$\ddagger$ A minimal curve is a curve (always imaginary) of zero length: $d x_{1}^{2}+d x_{2}^{2}+d x_{3}^{2}=0$.

$\S$ W. Blaschke, Vorlesungen über Differentialgeometrie, Berlin, 1921, vol. 1, p. 30. 


$$
\begin{aligned}
& x_{1}=i\left(f-t f^{\prime}-\frac{1-t^{2}}{2} f^{\prime \prime}\right), \\
& x_{2}=f-t f^{\prime}+\frac{1+t^{2}}{2} f^{\prime \prime}, \\
& x_{3}=-i\left(f^{\prime}-t f^{\prime \prime}\right) .
\end{aligned}
$$

Another point of view as to double minimal surfaces is contained in the formulas of Weierstrass for a (real) minimal surface:

$$
\begin{aligned}
& x_{1}=\Re \int\left(1-u^{2}\right) F(u) d u, \\
& x_{2}=\Re \int i\left(1+u^{2}\right) F(u) d u, \\
& x_{3}=\Re \int 2 u F(u) d u .
\end{aligned}
$$

The same surface will be represented if we use instead of $F(u)$ the function

$$
G(u)=-\frac{1}{u^{4}} \bar{F}\left(-\frac{1}{u}\right),
$$

the bar denoting the conjugate complex function. In fact, $F$ and $G$ correspond to the two different modes of generation of the minimal surface by translation of a minimal curve (the second minimal curve may be translated along the first as well as the first along the second). The condition for a double minimal surface is that these two modes of representation be identical:

$$
F(u)=-\frac{1}{u^{4}} \bar{F}\left(-\frac{1}{u}\right) .
$$

Then, unless the surface is periodic, it will be one-sided in the topological sense.

A simple example of a one-sided minimal surface was given by L. Henneberg* and is named after him; it is defined by

$$
F(u)=1-\frac{1}{u^{4}}
$$

easily verified to satisfy the condition (1.1).

* L. Henneberg, Ueber solche Minimalfächen die eine vorgeschriebene ebene Curve zur geodätischen Linie haben, Zurich, 1875. 
For further information as to one-sided minimal surfaces the reader is referred to the treatises of Darboux* and Scheffers. $\dagger$

\section{The problem of Plateau for one-sided minimal surfaces}

The purpose of the present paper is to solve the problem of Plateau for one-sided minimal surfaces: given a closed contour $\Gamma$, to prove, under appropriate sufficient conditions, the existence of a one-sided minimal surface bounded by $\Gamma$.

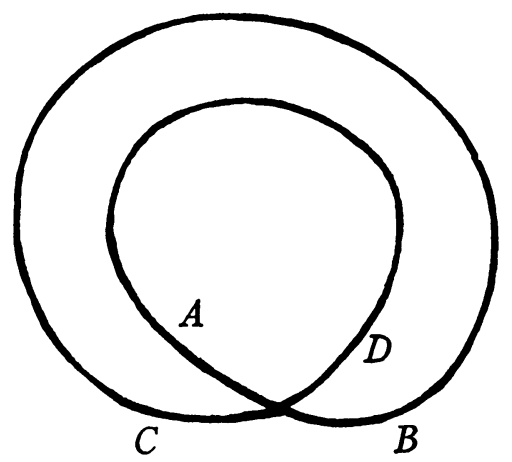

Fig. 1

Contrary to what one might surmise off-hand, no very complicated contour is required in order to get a one-sided minimal surface. If the soap-film experiment is performed with the contour indicated in the figure (where the double point is only apparent, the $\operatorname{arc} A B$ passing above the $\operatorname{arc} C D$ ) then the film obtained will be topologically equivalent to a Möbius strip, whose form the reader will find easy to visualize. Certainly, there also exists a minimal surface bounded by the same contour having the ordinary topology of a circular disc; but this other surface is self-intersecting, and it is rather the one-sided surface which is obtained in the actual experiment.

The present writer introduced into the Plateau problem a certain functional $A(g)$, by means of which were given the first general solution of the problem for the case of a single contour $\ddagger$ and for the case of two contours. $\$$ The governing thought of the present paper is that the problem of a one-sided minimal surface bounded by a given contour may be regarded as a limit form

* Leçons sur la Théorie Générale des Surfaces, Paris, 1887, pp. 340-364.

$\dagger$ Einführung in die Theorie der Flächen, Leipzig, 1902, pp. 256-260.

$\ddagger$ J. Douglas, Solution of the problem of Plateau, these Transactions, vol. 33, No. 1 (January, 1931), pp. 263-321.

This paper will be cited hereafter as One Contour.

$\S$ J. Douglas, The problem of Platean for tw'o contours, Journal of Mathematics and Physics of the Massachusetts Institute of Technology, vol. 10, No. 4 (December, 1931), pp. 315-359.

This paper will be cited hereafter as Tw'o Contours. 
of the two-contour case when the two contours become coincident. This may be seen by imagining a two-sided covering surface laid over the one-sided surface; the covering surface has two boundaries coinciding in $\Gamma$. The present paper follows out this idea, adapting the methods and formulas of the twocontour case to give an existence theorem for the one-sided minimal surface.

In the one-contour case the minimal surface was represented as topological image of a circular disc, in the two-contour case as image of a circular ring. It is necessary to have a normal region on which any surface homeomorphic to a Möbius strip can be represented topologically. Such a region is obtained by taking a ring bounded by two concentric circles $|z|=1,|z|=q$ $(q<1)$, and regarding as identical with one another any two points of the ring elliptically inverse with respect to the concentric circle of radius $q^{1 / 2}$; we may also say that we consider as a single element the pair of points $\left(z,-q / z_{0}\right)$ (notation: the subscript zero is used systematically in this paper to denote the conjugate complex quantity). The point $-q / z_{0}$ is the symmetrical image in the origin of the point inverse to $z$ with respect to the circle of radius $q^{1 / 2}$ (meaning of "elliptically inverse").

The two points thus associated in a pair will be termed equivalent.

If the ring be projected stereographically on the sphere having the circle of radius $q^{1 / 2}$ for equator, then the ring becomes a zone of the sphere, on which equivalent points become diametrically opposite points. The sphere with diametrically opposite points regarded as identical is a well known example of a one-sided manifold, and the zone upon it is topologically a Möbius strip.

A one-sided surface will be represented by writing the space coördinates $x_{i}$ of an arbitrary point equal to real-valued continuous (not at all monogenic) functions $x_{i}=f_{i}(w)$, of the complex variable $w$ ranging over the circular ring (or spherical zone), provided the functions $f_{i}$ take the same value for $w$ and $-q / w_{0}$.

The functional $A(g ; q)$; definitions of $m(\Gamma)$ and $\bar{m}(\Gamma)$. Given the contour $\Gamma$, we can associate with it two positive numbers $m(\Gamma), \bar{m}(\Gamma)$; the first pertains to $\Gamma$ regarded as boundary of surfaces topologically equivalent to a circular disc, the second arises from considering $\Gamma$ as boundary of surfaces of the topological type of a Möbius strip.

In the one-contour case we defined

$$
m(\Gamma)=\min A(g) .
$$

The functional $A(g)$ depends on an arbitrary parametric representation of $\Gamma$ :

$$
x_{i}=g_{i}(z)
$$

where the complex variable $z$ ranges over the unit circumference. If $\Re F_{i}(w)$ 
is the harmonic function on the unit circular disc determined by the boundary values $g_{i}(z)$, then

$$
x_{i}=\Re F_{i}(w)
$$

defines a harmonic surface bounded by $\Gamma$. Let the first fundamental form of this surface be

$$
d s^{2}=E d u^{2}+2 F d u d v+G d v^{2}
$$

then, by definition,

$$
A(g)=\iint \frac{1}{2}(E+G) d \sigma,
$$

$d \sigma$ being the element of area of the circular disc, over which the double integral is to be taken. With the help of Green's theorem, the more explicit formula was obtained:*

$$
A(g)=\frac{1}{4 \pi} \int_{C} \int_{C} \sum_{i=1}^{n}\left[g_{i}(z)-g_{i}(\zeta)\right]^{2} \frac{d z d \zeta}{(z-\zeta)^{2}}
$$

where $z, \zeta$ vary independently over the unit circumference $C$.

For the case of two contours the generalization of $A(g)$ is

where

$$
\begin{aligned}
& A\left(g_{1}, g_{2} ; q\right)=\iint \frac{1}{2}(E+G) d \sigma \\
&=\frac{1}{4 \pi} \sum_{\alpha \beta} \int_{C_{\alpha}} \int_{C_{\beta}} \sum_{i=1}^{n}\left[g_{\alpha i}(z)-g_{\beta i}(\zeta)\right]^{2} P(z, \zeta ; q) d z d \zeta \\
&(\alpha, \beta=1,2),
\end{aligned}
$$

$$
P(z, \zeta ; q)=-\frac{1}{2 \log q} \frac{1}{z \zeta}+\sum_{m=-\infty}^{\infty} \frac{q^{2 m}}{\left(q^{2 m} z-\zeta\right)^{2}}=P(\zeta, z ; q) .
$$

Here the two contours $\Gamma_{1}, \Gamma_{2}$ are represented parametrically in the form

$$
x_{i}=g_{1 i}(z), \quad x_{i}=g_{2 i}(z)
$$

where $z$ moves respectively over the circumferences of the circles $C_{1}(|z|=1)$ and $C_{2}(|z|=q)$ in the sense which keeps the interior of the ring bounded by these circles on the left. $E, F, G$ are the fundamental quantities of the doubly connected harmonic surface determined by the boundary values (2.7), and the integration in (2.4) is over the ring $C_{1} C_{2}$.

* Two Contours, formula 3.8. 
The identification of equivalent points of the circular ring enables us to pass directly from the two-contour case to that of a one-sided surface. If $z$ is on the unit circumference, then $z_{0}{ }^{\circ}=1 / z$ and therefore the equivalent point is $z^{\prime}=-q / z_{0}=-q z$, then $z=-z^{\prime} / q$, hence

$$
g_{2 i}(z)=g_{1 i}\left(-\frac{z}{q}\right) .
$$

In terms of the polar angle $\theta$,

$$
g_{2}(\theta)=g_{1}(\theta+\pi) .
$$

If, then, the contour $\Gamma$ is represented parametrically by reference to the unit circle in the form

$$
x_{i}=g_{i}(z),
$$

the representation of $\Gamma$ as image of the inner circle $C_{2}$ of the ring is determined:

$$
x_{i}=g_{i}\left(-\frac{z}{q}\right) .
$$

(2.10) and (2.11), taken as boundary values, determine a harmonic vector function in the circular ring, which evidently takes identical values at equivalent points, since the elliptical inversion of the ring into itself converts the boundary values into themselves, and also converts a harmonic function into a harmonic function; it need only be remarked in addition that a harmonic function is uniquely determined by its boundary values.

This harmonic vector function therefore defines a one-sided harmonic surface bounded by $\Gamma$, and now the natural definition for the functional $A$ is

$$
A(g ; q)=\frac{1}{2} \iint \frac{1}{2}(E+G) d \sigma,
$$

where the integration is over the circular ring, and the additional $\frac{1}{2}$ factor is meant to allow for the fact that the surface is obtained twice, each point with its antipodal, when the element $d \sigma$ varies over the whole circular ring.

This definition is equivalent to

$$
A(g ; q)=\frac{1}{2} A[g(\theta), g(\theta+\pi) ; q],
$$

the $A$ of the two vector arguments and $q$ referring to two contours.

To obtain $A(g ; q)$ explicitly in terms of its arguments: $g$ the variable parametric representation of $\Gamma$, and $q$ the real parameter $>0,<1$, we make the substitution (2.8) in the formula (2.5) for $A\left(g_{1}, g_{2} ; q\right)$, and find, after some 
reduction,

$$
A(g ; q)=\frac{1}{4 \pi} \int_{C} \int_{C} \sum_{i=1}^{n}\left[g_{i}(z)-g_{i}(\zeta)\right]^{2} Q(z, \zeta ; q) d z d \zeta
$$

where (the different senses in which $C_{1}$ and $C_{2}$ are described must be taken into account)

$$
Q(z, \zeta ; q)=P(z, \zeta ; q)+q P(z,-q \zeta ; q)
$$

or, by (2.6),

$$
Q(z, \zeta ; q)=\sum_{m=-\infty}^{\infty} \frac{q^{m}}{\left[q^{m} z-(-1)^{m} \zeta\right]^{2}}=\sum_{m=-\infty}^{\infty} \frac{q^{m}}{\left[z-(-1)^{m} q^{m} \zeta\right]^{2}}
$$

$A(g ; q)$, as defined by $(2.14)$, is the fundamental functional for the theory of minimal surfaces of the topological type of a Möbius strip. The function $Q$ which figures in its expression amounts essentially to an elliptic function of periods $2 \pi, 2 i \log q$.

We can now define, analogous to (2.1),

$$
\bar{m}(\Gamma)=\min A(g ; q),
$$

where the minimum is with respect to all parametric representations $g$ of $\Gamma$ and all values of $q, 0<q<1$.

Concretely, $m(\Gamma)$ is the minimum area of all surfaces homeomorphic to a circular disc which are bounded by $\Gamma$, and $\bar{m}(\Gamma)$ the minimum area of all surfaces homeomorphic to a Möbius strip which are bounded by $\Gamma$. Now, although the minimal surfaces obtained in this paper will, as a matter of fact, have the least area of all surfaces of the same topology bounded by $\Gamma$, we shall not be concerned primarily with this least-area aspect, taking rather the Weierstrass formulas:

$$
x_{i}=\Re F_{i}(w), \sum_{i=1}^{n} F_{i}^{\prime 2}(w)=0,
$$

as definition of a minimal surface. For us, therefore, $m(\Gamma)$ and $\bar{m}(\Gamma)$ are certain definite positive real numbers determined by the contour $\Gamma$ according to the definitions (2.1), (2.16).

If $q=0$, the formulas (2.14), (2.15) give

$$
A(g ; 0)=\frac{1}{4 \pi} \int_{C} \int_{C} \sum_{i=1}^{n}\left[g_{i}(z)-g_{i}(\zeta)\right]^{2} \frac{d z d \zeta}{(z-\zeta)^{2}}=A(g)
$$

From this we conclude that always

$$
\bar{m}(\Gamma) \leqq m(\Gamma)
$$


Suppose that we are in a case where the strict inequality holds:

$$
\bar{m}(\Gamma)<m(\Gamma)
$$

this happens, for instance, with the contour in Fig. 1. Then we are in a position to state the main theorem of this paper.

TheOREM I. Let $\Gamma$ denote any Jordan curve in euclidean space of $n$ dimensions with $m(\Gamma)$. finite and $\bar{m}(\Gamma)<m(\Gamma)$.

Then there exists a ring bounded by two concentric circles

$$
|z|=1,|z|=q, 0<q<1,
$$

and $n$ functions $F_{i}(w)$ of the complex variable w having the following properties:

(i) $F_{i}(w)$ is holomorphic in the circular ring;

$$
\begin{gathered}
F_{i}\left(-\frac{q}{w_{0}}\right)=F_{i}(w) ; \\
\sum_{i=1}^{n} F_{i}^{\prime 2}(w)=0 ;
\end{gathered}
$$

(iv) $x_{i}=\Re F_{i}(w)$ attaches continuously to boundary values

$$
\begin{array}{ll}
x_{i}=g_{i}(\theta) \text { on }|z|=1, & z=e^{i \theta}, \\
x_{i}=g_{i}(\theta+\pi) \text { on }|z|=q, & z=q e^{i \theta},
\end{array}
$$

each of which is a parametric representation of $\Gamma$.

In other words, the equations $x_{i}=\Re F_{i}(w)$ represent a one-sided minimal surface bounded by $\Gamma$.

The area of this surface is equal to $\bar{m}(\Gamma)$.

An interesting new theorem concerning one-sided minimal surfaces will be proved in $\$ 6$ as a corollary of this main theorem. It is

THEOREM II. Let $\Gamma$ be any contour, and suppose the minimal surface $M$ of the topological type of a circular disc which is determined by $\Gamma$ to have in its interior a singular point (branch point) of even order, i.e. a point where (the equations of $M$ being $x_{i}=\Re F_{i}(w)$ )

$$
\begin{gathered}
\sum_{i=1}^{n}\left|F_{i}^{\prime}(w)\right|^{2}=0, \quad \sum_{i=1}^{n}\left|F_{i}^{\prime \prime}(w)\right|^{2}=0, \cdots, \sum_{i=1}^{n}\left|F^{(2 k-1)}(w)\right|^{2}=0, \\
\sum_{i=1}^{n}\left|F^{(2 k)}(w)\right|^{2}>0 .
\end{gathered}
$$

Then there exists a one-sided minimal surface $\bar{M}$ bounded by $\Gamma$. 
The area of $\bar{M}$ is less than the area of $M$ (unless the area of $M$ is infinite; then so is the area of $\bar{M}$ ).

It is this theorem which would assure us a priori of the existence of a onesided minimal surface bounded by the contour shown in Fig. 1. For the surface $M$ determined by that contour is readily visualized to have the general form of the Riemann surface for $z^{1 / 2}$, with a branch point of second order in its interior.

\section{The minimizing Representation of $A(g ; q)$}

To represent the contour $\Gamma$ we are using two concentric circles $C$ and $C_{q}$ of radii 1 and $q<1$. Let $\theta$ denote the polar angle on each circle; then if $x_{i}=g_{i}(\theta)$ is a parametric representation of $\Gamma$ as topological image of $C$, its representation as image of $C_{q}$ will be determined as $x_{i}=g_{i}(\theta+\pi)$. Thus a representation of $\Gamma$ is completely determined by the vector function $g$ and the value of $q:[g ; q]$.

Now, we have seen in my previous papers on the problem of Plateau that the totality of parametric representations $g$ of $\Gamma$ is a compact Fréchet $L$-set, provided we include certain exceptional representations called improper and degenerate according to the following definitions:

(i) Improper of the first kind: an arc of $\Gamma$ less than all of $\Gamma$ corresponds to a point of $C$.

(ii) Improper of the second kind: an $\operatorname{arc}$ of $C$ less than all of $C$ corresponds to a point of $\Gamma$.

(iii) Degenerate: all of $C$ corresponds to a point of $\Gamma$, and all of $\Gamma$ to a point of $C$.

The range of values of $q$ is compact provided we include the extreme values 0 and 1 .

Therefore, with the inclusion of these exceptional forms of $g$ and values of $q$, the composite elements $[g ; q]$ form a compact closed set.

On this set $A(g ; q)$ is a lower semi-continuous functional. For, by the form of definition (2.12) of $A(g ; q)$,

$$
A(g ; q)=\frac{1}{2} \iint \frac{1}{2}(E+G) d \sigma,
$$

where the domain of integration is the ring $R$ between $C$ and $C_{q}$. If instead, we take the ring $R^{\prime}$ between $C^{\prime}$ and $C_{q}^{\prime}$ of radii $1-\epsilon$ and $q /(1-\epsilon)$ respectively, then the resulting functional $A_{\epsilon}(g ; q)$ has a uniformly bounded integrand; consequently it is a continuous functional by the theorem that one may pass to the limit under the sign of integration if the integrand stays uniformly 
bounded. By definition,

$$
A(g ; q)=\lim _{\epsilon \rightarrow 0} A_{\epsilon}(g ; q),
$$

and the approach is always in the increasing sense, for diminishing the value of $\epsilon$ adds positive elements

$$
\frac{1}{2}(E+G) d \sigma=\frac{1}{2} \sum_{i=1}^{n}\left[\left(\frac{\partial x_{i}}{\partial u}\right)^{2}+\left(\frac{\partial x_{i}}{\partial v}\right)^{2}\right] d u d v
$$

to the integral. Now the following is an easily proved theorem: $\dagger$ if $A, a$ functional on any Fréchet $L$-set, can be represented as the limit of a continuous functional on that set which approaches to $A$ in increasing, then $A$ is lower semicontinuous. Hence the stated lower semi-continuity of $A(g ; q)$.

In Fréchet's thesis $\ddagger$ it is easily shown that a lower semi-continuous functional on a compact set attains its minimum value. Therefore, there exists a representation $\left[g^{*} ; q^{*}\right]$ of $\Gamma$ for which the minimum value $\bar{m}(\Gamma)$ of $A(g ; q)$ is attained.

Exclusion of improper representations. However, to secure the compactness of the set $[g ; q]$ we have found it necessary to adjoin certain improper representations. We have next to exclude the possibility that it is for one of these improper representations that the minimum of $A(g ; q)$ is attained.

First, suppose $q \neq 0, \neq 1$. Then, if $g$ is improper of type (i),

$$
A(g ; q)=+\infty \text {. }
$$

For if the expression (2.14) for $A(g ; q)$ be expanded according to the formula (2.15) for $Q(z, \zeta ; q)$, the expansion contains for $m=0$ the term

$$
\frac{1}{4 \pi} \int_{C} \int_{C} \sum_{i=1}^{n}\left[g_{i}(z)-g_{i}(\zeta)\right]^{2} \frac{d z d \zeta}{(z-\zeta)^{2}}
$$

and if $l>0$ is the length of the chord of the arc of $\Gamma$ that corresponds to the point $P$ of $C$, then for $z$ and $\zeta$ near to $P$ this integral has asymptotically the form

$$
\iint l^{2} \frac{d z d \zeta}{(z-\zeta)^{2}}
$$

The order to which the integrand becomes infinite for $z=\zeta$ secures the result (3.1), the indefinite integral being $l^{2} \log (z-\zeta)$.

Therefore the minimizing representation of $A(g ; q)$ cannot be improper of the first kind.

$\dagger$ One Contour, p. 282.

pp. 1-74.

$\ddagger$ M. Fréchet, Sur quelques points du calcul fonctionnel, Rendiconti di Palermo, vol. 22 (1906), 
Next, it cannot be degenerate, type (ii). We prolong the definition of $A(g ; q)$ to degenerate representations by lower semi-continuity: if $g_{d}$ denote a degenerate representation,

$$
A\left(g_{d} ; q\right)=\lim \inf A(g ; q) \text { for } g \rightarrow g_{d} .
$$

Returning to the formula (2.14) as expanded by (2.15), we see, since the vector function $g_{d}$ is a constant (reduces to a point), that every term $m \neq 0$ vanishes, while the term $m=0$ is simply (3.2) or $A(g)$, whose inferior limit for $g \rightarrow g_{d}$ is $m(\Gamma)$. Hence

$$
A\left(g_{d} ; q\right)=m(\Gamma) \quad(0<q<1) .
$$

But by the basic hypothesis, $m(\Gamma)>\bar{m}(\Gamma)$. Hence the representation for which the minimum $\bar{m}(\Gamma)$ is attained is certainly not degenerate.

Consider next the case $q=0$. We have immediately, by (2.14), (2.15) and (2.3),

$$
A(g ; 0)=A(g),
$$

and for every $g, A(g) \geqq m(\Gamma)>\bar{m}(\Gamma)$. The possibility of a minimum occurring for $q=0$ is thus eliminated.

Finally, we have to consider the possibility $q=1$. If $q$ becomes 1 in the formulas (2.14), (2.15), then each term approaches the positive real quantity $A(g)$, provided $g$ does not at the same time become degenerate. Hence, for $g$ non-degenerate,

$$
A(g ; 1)=+\infty \quad\left(g \neq g_{d}\right) .
$$

On the other hand, if $g$ stays degenerate, $g_{d}$, while $q \rightarrow 1$, then it will be seen from (3.3) that $A(g ; q)$ stays constantly equal to $m(\Gamma)$, and this is the least limit that can be approached by $A(g ; q)$ if simultaneously $g \rightarrow g_{d}$ and $q \rightarrow 1$. By lower semi-continuity, then,

$$
A\left(g_{d} ; 1\right)=m(\Gamma) .
$$

(3.5) and (3.6) show that the minimum $\bar{m}(\Gamma)$ cannot be attained for $q=1$.

Of all the improper representations, there remains now only type (ii) with $0<q<1$. We shall not be able to exclude this, and so make certain that the minimizing representation of $A(g ; q)$ is proper, until we have proved in the next section that the vanishing of the first variation of $A(g ; q)$ :

$$
\delta A(g ; q)=0,
$$

leads to the condition for a minimal surface:

$$
\sum_{i=1}^{n} F_{i}^{\prime 2}(w)=0 .
$$




\section{VANISHING OF THE FIRST VARIATION OF $A(g ; q)$}

We have now established the existence of a minimizing representation $\left[g^{*} ; q^{*}\right]$ of $A(g ; q)$ :

$$
A\left(g^{*} ; q^{*}\right)=\bar{m}(\Gamma)
$$

where $0<q^{*}<1$ and $g^{*}$ is proper, or possibly (what will later be excluded) improper in the manner that $g^{*}$ stays constant on certain arcs of $C$ (in either event $g^{*}$ is continuous).

The next step is to express the vanishing of the first variation of $A(g ; q)$ for $\left[g^{*} ; q^{*}\right]$.

To this end we use the methoc known in the calculus of variations as "variation of the independent variables." In fact, the following variation of $z$ and $\zeta$ will lead most elegantly to the desired result:

$$
z^{\prime}=z+\lambda z^{2} V(z, w ; q)
$$

where $\lambda$ is a real parameter, $w$ any point interior to the ring $C, C_{q}(q<|w|<1)$, and $V(z, w ; q)$ is the following function:

$$
\begin{aligned}
V(z, w ; q)= & \sum_{m=0}^{\infty} \frac{(-1)^{m} q^{m}}{(-1)^{m} q^{m} z-w} \\
& +\sum_{m=1}^{\infty}\left\{\frac{1}{z-(-1)^{m} q^{m} w}-\frac{1}{z}\right\} .
\end{aligned}
$$

Justification of the special variation. The introduction of the variation (4.1) necessitates the following remarks.

The only kind of variation of the independent variable $z$ that we have the right to use is one which converts the unit circle into itself in a monotonic continuous way; we imagine that the attached value of the vector function $g(z)$ is transported along with $z$, and so we pass from one representation of $\Gamma$ as topological image of $C$ to another.

Now the variation which has been proposed above does not convert the unit circle $C$ into itself at all, let alone in a monotonic continuous way. Its use therefore requires justification.

Let us, as usual, employ the subscript zero to denote the conjugate complex quantity; then if $\lambda$ is a real parameter, evidently

$$
\lambda\left\{z V(z, w ; q)-z_{0} V\left(z_{0}, w_{0} ; q\right)\right\}
$$

and

$$
i \lambda\left\{z V(z, w ; q)+z_{0} V\left(z_{0}, w_{0} ; q\right)\right\}
$$


are pure imaginary quantities. Therefore the exponential of each of these quantities defines a rotation about the origin through an angle which is a function of $z$ (w fixed); consequently the transformations

$$
\begin{aligned}
& z^{\prime}=z \exp \left[\lambda\left\{V(z, w ; q)-z_{0} V\left(z_{0}, w_{0} ; q\right)\right\}\right], \\
& z^{\prime}=z \exp \left[i \lambda\left\{z V(z, w ; q)+z_{0} V\left(z_{0}, w_{0} ; q\right)\right\}\right]
\end{aligned}
$$

convert a point on the unit circumference into a point on the unit circumference.

Furthermore, for $\lambda=0$ these transformations reduce to the identity, for which

$$
\frac{d \theta^{\prime}}{d \theta}=\frac{d \log z^{\prime}}{d \log z}=1 \quad\left(z=e^{i \theta}\right) .
$$

Now $d \log z^{\prime} / d \log z$ is a function which is regular except when one of the denominators in the expression (4.2) for $V(z, w ; q)$ vanishes. But by the restriction on the fixed point $w$,

$$
q<|w|<1,
$$

these denominators stay uniformly bounded away from zero when $z$ describes $C$; hence for any fixed $w$ subject to (4.4), all $z$ on $C$, and all $\lambda$ sufficiently near to zero, we have

$$
\frac{d \theta^{\prime}}{d \theta} \text { nearly }=1, \text { therefore certainly }>0 .
$$

Consequently for any fixed $w$ interior to the ring (4.4), and all sufficiently small $\lambda$ of either sign (the smallness of $\lambda$ depending on the position of $w$ ), the transformations (4.3) are monotonic continuous of the unit circumference into itself.

If we apply, say, the first transformation, $\left(4.3_{1}\right)$, to $\left[g^{*} ; q^{*}\right]$, obtaining $\left[g_{\lambda}{ }^{*} ; q^{*}\right]$, then the value of $A\left(g_{\lambda}^{*} ; q^{*}\right)$ is a function of $\lambda, A_{1}(\lambda)$, with a minimum at $\lambda=0$ relative to some interval about that point. If, therefore, $A_{1}(\lambda)$ be expanded in powers of $\lambda$, as can be done: $\dagger$

$$
A_{1}(\lambda)=A\left(g^{*} ; q^{*}\right)+\lambda V_{1}+\cdots,
$$

then

$$
V_{1}=0
$$

† A detailed function-theoretic justification, based on classical convergence theorems, is in One Contour, $\$ \$ 13,14$. 
Similarly, with the use of $\left(4.3_{2}\right)$, we have

$$
A_{2}(\lambda)=A\left(g^{*} ; q^{*}\right)+i \lambda V_{2}+\cdots,
$$

whence

$$
V_{2}=0 .
$$

The expansions $\left(4.6_{1}\right),\left(4.6_{2}\right)$ depend on the expansions in powers of $\lambda$ of the transformations $\left(4.3_{1}\right),\left(4.3_{2}\right)$ :

$$
\begin{aligned}
& z^{\prime}=z+\lambda\left\{z^{2} V(z, w ; q)-z z_{0} V\left(z_{0}, w_{0} ; q\right)\right\}+\cdots, \\
& z^{\prime}=z+i \lambda\left\{z^{2} V(z, w ; q)+z z_{0} V\left(z_{0}, w_{0} ; q\right)\right\}+\cdots .
\end{aligned}
$$

It is evident that the values $V_{1}, V_{2}$ obtained for the coefficient of $\lambda$ in the expansions $\left(4.6_{1}\right),\left(4.6_{2}\right)$ would be the same if we kept only the linear part of the expansions $\left(4.8_{1}\right),\left(4.8_{2}\right)$ :

$$
\begin{aligned}
& z^{\prime}=z+\lambda\left\{z^{2} V(z, w ; q)-z z_{0} V\left(z_{0}, w_{0} ; q\right)\right\}, \\
& z^{\prime}=z+i \lambda\left\{z^{2} V(z, w ; q)+z z_{0} V\left(z_{0}, w_{0} ; q\right)\right\} .
\end{aligned}
$$

And now if we use the variation

$$
(4.10)=(4.1)
$$$$
z^{\prime}=z+\lambda z^{2} V(z, w ; q),
$$

formed by taking as coefficient of the parameter $\lambda$ one-half the sum of the corresponding coefficients in $\left(4.9_{1}\right),\left(4.9_{2}\right)$, it is evident that we shall get on expanding $A\left(g_{\lambda}^{*} ; q^{*}\right)$

$$
A\left(g^{*} ; q^{*}\right)+\lambda \frac{V_{1}+V_{2}}{2}+\cdots,
$$

and from $\left(4.7_{1}\right)$ and $\left(4.7_{2}\right)$ it follows that we have the right to put the coefficient of $\lambda$ so obtained equal to zero. $\dagger$

The use of the variation (4.10) being thus proved permissible, we next apply it, as well as the same transformation in $\zeta$,

$$
\zeta^{\prime}=\zeta+\lambda \zeta^{2} V(\zeta, w ; q)
$$

to the functional $A(g ; q)$ (formula 2.14) with

$$
[g ; q]=\left[g^{*} ; q^{*}\right]
$$

then expanding in powers of $\lambda$ and annulling the coefficient of the first power of $\lambda$, we have (where through the rest of this section the substitution (4.13) will be understood)

† The above argument is the same as that used in Two Contours, pp. 332-334. 


$$
\begin{aligned}
0= & \int_{C} \int_{C} \sum_{i=1}^{n}\left[g_{i}(z)-g_{i}(\zeta)\right]^{2} \\
& \cdot\left[z^{2} Q_{z}(z, \zeta ; q) V(z, w ; q)+\zeta^{2} Q_{\zeta}(z, \zeta ; q) V(\zeta, w ; q)\right. \\
& +2 z Q(z, \zeta ; q) V(z, w ; q)+z^{2} Q(z, \zeta ; q) V_{z}(z, w ; q) \\
& \left.+2 \zeta Q(z, \zeta ; q) V(\zeta, w ; q)+\zeta^{2} Q(z, \zeta ; q) V_{\zeta}(\zeta, w ; q)\right] d z d \zeta
\end{aligned}
$$

The function $Q(z, \zeta ; q)$ is evidently homogeneous of the minus second degree in $z, \zeta$, so that we have Euler's equation:

$$
z Q_{z}(z, \zeta ; q)+\zeta Q_{\zeta}(z, \zeta ; q)+2 Q(z, \zeta ; q)=0
$$

with whose help the condition (4.14) simplifies to

$$
\begin{aligned}
0= & \int_{C} \int_{C} \sum_{i=1}^{n}\left[g_{i}(z)-g_{i}(\zeta)\right]^{2} \\
& \cdot\left[z^{2} Q(z, \zeta ; q) V_{z}(z, w ; q)+\zeta^{2} Q(z, \zeta ; q) V_{\zeta}(\zeta, w ; q)\right. \\
& \left.-z \zeta Q_{\zeta}(z, \zeta ; q) V(z, w ; q)-z \zeta Q_{z}(z, \zeta ; q) V(\zeta, w ; q)\right] d z d \zeta .
\end{aligned}
$$

Now the variation of $A(g ; q)$ is also zero when we vary $q$ away from $q^{*}$, keeping $g^{*}$ fixed; i.e.

$$
\frac{\partial A(g ; q)}{\partial q}=0
$$

or

$$
0=\int_{C} \int_{C} \sum_{i=1}^{n}\left[g_{i}(z)-g_{i}(\zeta)\right]^{2} \cdot \frac{\partial}{\partial q} Q(z, \zeta ; q) d z d \zeta,
$$

for $[g ; q]=\left[g^{*} ; q^{*}\right]$.

At this point, we need to make use of the following identity governing the functions $V$ and $Q$ :

$$
w^{2} Q(z, w ; q) Q(\zeta, w ; q)=-z^{2} Q(z, \zeta ; q) V_{z}(z, w ; q)
$$

$$
\begin{aligned}
& -\zeta^{2} Q(z, \zeta ; q) V_{\zeta}(\zeta, w ; q)+z \zeta Q_{\zeta}(z, \zeta ; q) V(z, w ; q) \\
& +z \zeta Q_{z}(z, \zeta ; q) V(\zeta, w ; q)+q \frac{\partial}{\partial q} Q(z, \zeta ; q)
\end{aligned}
$$

$V$ and $Q$ are essentially elliptic functions with periods $2 \pi, 2 i \log q$, and the identity is tantamount to the Weierstrass partial differential equation for $\wp\left(u ; \omega_{1}, \omega_{2}\right)$ considered as a function of all three arguments. In order not to interrupt the thread of argument, the consequences of this identity will be drawn immediately, and its proof postponed to the next section. 
If we multiply (4.16) by $-1,(4.18)$ by $q$, and add, then we get as factor under the integral sign precisely the second member of the identity (4.19); replacing this by the first member and dividing out $w^{2}$, we obtain

$$
\int_{C} \int_{C} \sum_{i=1}^{n}\left[g_{i}(z)-g_{i}(\zeta)\right]^{2} Q(z, w ; q) Q(\zeta, w ; q) d z d \zeta=0 .
$$

This we readily show to be the same as the condition for a minimal surface:

$$
\sum_{i=1}^{n} F_{i}^{\prime 2}(w)=0
$$

as follows.

Let $x_{i}=\Re F_{i}(w)$ be the one-sided harmonic surface determined by the representation $[g ; q]$; the formula for $F_{i}(w)$ is easily found from the corresponding formula in Two Contours $\dagger$ by putting

it is

$$
g_{1}(z)=g(z), g_{2}(z)=g\left(-\frac{z}{q}\right) ;
$$

$$
F_{i}(w)=\frac{1}{\pi i} \int_{C} g_{i}(z) Y(z, w ; q) d z+c_{i},
$$

where

$$
\begin{aligned}
Y(z, w ; q)= & \sum_{m=0}^{\infty} \frac{q^{m}}{(-1)^{m} q^{m} z-w} \\
& +\sum_{m=1}^{\infty}(-1)^{m}\left\{\frac{1}{z-(-1)^{m} q^{m} w}-\frac{1}{z}\right\}, \\
c_{i}= & -\frac{1}{2 \pi i} \int_{C} g_{i}(z) \frac{d z}{z}=\frac{1}{2 \pi} \int_{C} g_{i}(\theta) d \theta .
\end{aligned}
$$

The function $Q(z, w ; q)$ is exactly the derivative of $Y(z, w ; q)$ as to $w$ :

$$
Q(z, w ; q)=\frac{\partial}{\partial w} Y(z, w ; q)
$$

(verify by (2.15)), so that we have, by differentiation of (4.22),

$$
F_{i}^{\prime}(w)=\frac{1}{\pi i} \int_{C} g_{i}(z) Q(z, w ; q) d z .
$$

$\dagger$ Loc. cit., formulas 4.5 and 4.7 
Rewriting this as

$$
F_{i}^{\prime}(w)=\frac{1}{\pi i} \int_{C} g_{i}(\zeta) Q(\zeta, w ; q) d \zeta,
$$

and multiplying with the preceding, then summing for $i$ from 1 to $n$, we get

$$
\sum_{i=1}^{n} F_{i}^{\prime 2}(w)=-\frac{1}{\pi^{2}} \int_{C} \int_{C} \sum_{i=1}^{n} g_{i}(z) g_{i}(\zeta) Q(z, w ; q) Q(\zeta, w ; q) d z d \zeta
$$

The value of this is not changed if we write it

$$
\sum_{i=1}^{n} F_{i}^{\prime 2}(w)=\frac{1}{2 \pi^{2}} \int_{C} \int_{C} \sum_{i=1}^{n}\left[g_{i}(z)-g_{i}(\zeta)\right]^{2} Q(z, w ; q) Q(\zeta, w ; q) d z d \zeta
$$

the terms $g_{i}(z)^{2}, g_{i}(\zeta)^{2}$ contributing zero since

$$
\int_{C} Q(z, w ; q) d z=0, \int_{C} Q(\zeta, w ; q) d \zeta=0
$$

(the indefinite integral is a uniform function).

The comparison of (4.28) with (4.20) establishes the result (4.21): $\sum_{i=1}^{n}$ $F_{i}{ }^{2}(w)=0$.

Exclusion of improper representation, type (ii). Finally, the condition (4.21) enables us to exclude the one remaining possible type of improper representation, in which an arc of $C$ less than all of $C$ is made to correspond to a point of $\Gamma$. For (4.21) implies, with the help of a well known theorem of Fatou and the Schwarz symmetry principle, that $\Re F_{i}(w)$, for $i=1,2, \cdots, n$, cannot be constant on an arc of $C$ without being constant on all of $C$. The simple discussion was given in One Contour (p. 301) and need not be repeated here.

To sum up, we have proved the existence of a one-one continuous correspondence between $\Gamma$ and $C$, furnishing a parametric representation of $\Gamma$, $x_{i}=g_{i}^{*}(\theta)$, such that this together with $x_{i}=g_{i}^{*}(\theta+\pi)$ on a certain concentric circle of radius $q^{*}$ determines, when taken as boundary values, $n$ harmonic functions $x_{i}=\Re F_{i}(w)$ obeying the condition $\sum_{i=1}^{n} F_{i}{ }^{\prime 2}(w)=0$, and defining thus a minimal surface of the topological type of a Möbius strip bounded by $\Gamma$; the one-sidedness of the surface results from the condition $F_{i}\left(-q / w_{0}\right)$. $=F_{i}(w)$ implied by the relation between $g_{i}^{*}(\theta+\pi)$ and $g_{i}^{*}(\theta)$.

\section{Proof of the fundamental identity}

In this section we give the postponed proof of the identity (4.19) which played the decisive part in the argument of the last section. 
We start with the algebraic identity $\dagger$

$$
\begin{aligned}
& \frac{\partial}{\partial w}\left\{w^{2} \cdot \frac{q^{m}}{\left[w-(-1)^{m} q^{m} z\right]^{2}} \cdot \frac{q^{n}}{\left[w-(-1)^{n} q^{n} \zeta\right]^{2}}\right\} \\
& =-z^{2} \cdot \frac{q^{n-m}}{\left[z-(-1)^{n-m} q^{n-m} \zeta\right]^{2}} \cdot \frac{2 q^{2 m}}{\left[w-(-1)^{m} q^{m} z\right]^{3}} \\
& \quad-\zeta^{2} \cdot \frac{q^{n-m}}{\left[z-(-1)^{n-m} q^{n-m} \zeta\right]^{2}} \cdot \frac{2 q^{2 n}}{\left[w-(-1)^{n} q^{n} \zeta\right]^{3}} \\
& \quad+z \zeta \cdot \frac{2(-1)^{n-m} q^{2 n-2 m}}{\left[z-(-1)^{n-m} q^{n-m} \zeta\right]^{3}} \cdot \frac{(-1)^{m} q^{m}}{\left[w-(-1)^{m} q^{m} z\right]^{2}} \\
& \quad+z \zeta \cdot \frac{-2 q^{n-m}}{\left[z-(-1)^{n-m} q^{n-m} \zeta\right]^{3}} \cdot \frac{(-1)^{n} q^{n}}{\left[w-(-1)^{n} q^{n} \zeta\right]^{2}}
\end{aligned}
$$

whose verification is merely a matter of calculation. If the indices $m, n$ are made to vary independently from $-\infty$ to $+\infty$, then the pairs of indices $n-m, m$ and $n-m, n$ will do the same, so that by summation we have

$$
\begin{aligned}
& \frac{\partial}{\partial w}\left\{w^{2} \cdot \sum_{m=-\infty}^{\infty} \frac{q^{m}}{\left[w-(-1)^{m} q^{m} z\right]^{2}} \cdot \sum_{n=-\infty}^{\infty} \frac{q^{n}}{\left[w-(-1)^{n} q^{n} \zeta\right]^{2}}\right\} \\
& =-z^{2} \cdot \sum_{m=-\infty}^{\infty} \frac{q^{m}}{\left[z-(-1)^{m} q^{m} \zeta\right]^{2}} \cdot \sum_{n=-\infty}^{\infty} \frac{2 q^{2 n}}{\left[w-(-1)^{n} q^{n} z\right]^{3}} \\
& \quad-\zeta^{2} \cdot \sum_{m=-\infty}^{\infty} \frac{q^{m}}{\left[z-(-1)^{m} q^{m} \zeta\right]^{2}} \cdot \sum_{n=-\infty}^{\infty} \frac{2 q^{2 n}}{\left[w-(-1)^{n} q^{n} \zeta\right]^{3}} \\
& \quad+z \zeta \cdot \sum_{m=-\infty}^{\infty} \frac{2(-1)^{m} q^{2 m}}{\left[z-(-1)^{m} q^{m} \zeta\right]^{3}} \cdot \sum_{n=-\infty}^{\infty} \frac{(-1)^{n} q^{n}}{\left[w-(-1)^{n} q^{n} z\right]^{2}} \\
& \quad+z \zeta \cdot \sum_{m=-\infty}^{\infty} \frac{\left(-2 q^{m}\right.}{\left[z-(-1)^{m} q^{m} \zeta\right]^{3}} \cdot \sum_{n=-\infty}^{\infty} \frac{(-1)^{n} q^{n}}{\left[w-(-1)^{n} q^{n} \zeta\right]^{2}} .
\end{aligned}
$$

Rewriting the defining formulas of the functions $Q$ and $V$ :

$$
\begin{aligned}
\text { (5.3) } Q(z, \zeta ; q) & =\sum_{m=-\infty}^{\infty} \frac{q^{m}}{\left[z-(-1)^{m} q^{m} \zeta\right]^{2}}, \\
\text { (5.4) } V(z, w ; q) & =\sum_{m=0}^{\infty} \frac{(-1)^{m} q^{m}}{(-1)^{m} q^{m} z-w}+\sum_{m=1}^{\infty}\left\{\frac{1}{z-(-1)^{m} q^{m} w}-\frac{1}{z}\right\},
\end{aligned}
$$

we observe that the two-way infinite sums appearing in (5.2) are the same as

$\dagger$ Cf. Two Contours, last formula on page 346 , from which the present one can be derived by the following replacements: $2 m$ by $m, 2 n$ by $n, q$ by $-q$. 
these functions and their derivatives:

$$
\begin{aligned}
Q_{z}(z, \zeta ; q) & =\sum_{m=-\infty}^{\infty} \frac{-2 q^{m}}{\left[z-(-1)^{m} q^{m} \zeta\right]^{3}}, \\
Q_{\zeta}(z, \zeta ; q) & =\sum_{m=-\infty}^{\infty} \frac{2(-1)^{m} q^{2 m}}{\left[z-(-1)^{m} q^{m} \zeta\right]^{3}}, \\
V_{w}(z, w ; q) & =\sum_{m=-\infty}^{\infty} \frac{(-1)^{m} q^{m}}{\left[w-(-1)^{m} q^{m} z\right]^{2}}, \\
V_{z w}(z, w ; q) & =\sum_{m=-\infty}^{\infty} \frac{2 q^{2 m}}{\left[w-(-1)^{m} q^{m} z\right]^{3}}, \\
V_{\zeta w}(\zeta, w ; q) & =\sum_{m=-\infty}^{\infty} \frac{2 q^{2 m}}{\left[w-(-1)^{m} q^{m} \zeta\right]^{3}}
\end{aligned}
$$

so that, by substitution,

$$
\begin{aligned}
\frac{\partial}{\partial w}\left\{w^{2} Q(z, w ; q) Q(\zeta, w ; q)\right\} \\
=-z^{2} Q(z, \zeta ; q) V_{z w}(z, w ; q)-\zeta^{2} Q(z, \zeta ; q) V_{\zeta w}(\zeta, w ; q) \\
\quad+z \zeta Q_{\zeta}(z, \zeta ; q) V_{w}(z, w ; q)+z \zeta Q_{z}(z, \zeta ; q) V_{w}(\zeta, w ; q) .
\end{aligned}
$$

Integrating with respect to $w$,

$$
\begin{aligned}
& w^{2} Q(z, w ; q) Q(\zeta, w ; q) \\
& \quad=-z^{2} Q(z, \zeta ; q) V_{z}(z, w ; q)-\zeta^{2} Q(z, \zeta ; q) V_{\zeta}(\zeta, w ; q) \\
& \quad+z \zeta Q_{\zeta}(z, \zeta ; q) V(z, w ; q)+z \zeta Q_{z}(z, \zeta ; q) V(\zeta, w ; q)+\phi(z, \zeta ; q),
\end{aligned}
$$

where $\phi(z, \zeta ; q)$ is the constant of integration.

To determine $\phi(z, \zeta ; q)$ we expand each member in a Laurent series of $w$ and identify the terms free of $w$.

The Laurent expansion of $Q$ is

$$
\begin{aligned}
& Q(z, w ; q)=\frac{1}{z w} \sum_{m=-\infty}^{\infty} \frac{m}{1+(-1)^{m} q^{m}} \cdot \frac{z^{m}}{w^{m}}, \\
& Q(\zeta, w ; q)=\frac{1}{\zeta w} \sum_{m=-\infty}^{\infty} \frac{m}{1+(-1)^{m} q^{m}} \cdot \frac{\zeta^{m}}{w^{m}},
\end{aligned}
$$

from which it is easy to calculate that the term independent of $w$ in $w^{2} Q(z$, $w ; q) Q(\zeta, w ; q)$ is

$$
\frac{1}{z \zeta} \sum_{m=-\infty}^{\infty} \frac{-m^{2}(-1)^{m} q^{m}}{\left[1+(-1)^{m} q^{m}\right]^{2}} \cdot \frac{z^{m}}{\zeta^{m}}
$$


and this is recognized to be the same as

$$
q \frac{\partial}{\partial q} Q(z, \zeta ; q) \text {. }
$$

By (5.4), the term free of $w$ in the expansion of $V(z, w ; q), V(\zeta, w ; q)$, $V_{z}(z, w ; q), V_{\zeta}(\zeta, w ; q)$ is, respectively,

$$
\frac{1}{z}, \frac{1}{\zeta},-\frac{1}{z^{2}},-\frac{1}{\zeta^{2}}
$$

hence the term free of $w$ in the second member of (5.11) is

$$
\begin{aligned}
Q(z, \zeta ; q)+Q(z, \zeta ; q) & +\zeta Q_{\zeta}(z, \zeta ; q) \\
& +z Q_{z}(z, \zeta ; q)+\phi(z, \zeta ; q)
\end{aligned}
$$

But $Q(z, \zeta ; q)$ is homogeneous of the minus second degree in $z, \zeta$, so that, by Euler's equation,

$$
2 Q(z, \zeta ; q)+\zeta Q_{\zeta}(z, \zeta ; q)+z Q_{z}(z, \zeta ; q)=0
$$

therefore the comparison of the terms free of $w$ in the two members of (5.11) gives

$$
\phi(z, \zeta ; q)=q \frac{\partial}{\partial q} Q(z, \zeta ; q) .
$$

Substituting this in (5.11). we have finally the desired identity (4.19).

6. EFFECT OF SINGULAR POINTS OF THE TWO-SIDED MINIMAL SURFACE ON THE EXISTENCE OF THE ONE-SIDED MINIMAL SURFACE

In Two Contours the fundamental functional $A\left(g_{1}, g_{2} ; q\right)$ was expressed in terms of the Fourier coefficients of $g_{1}$ and $g_{2}: \dagger$

$$
\begin{aligned}
& A\left(g_{1}, g_{2} ; q\right)=\frac{\pi \alpha^{2}}{-\log q} \\
& +\frac{\pi}{2} \sum_{m=1}^{\infty} \frac{\left(1+q^{2 m}\right)\left(a_{1 m}^{2}+b_{1 m}^{2}+a_{2 m}^{2}+b_{2 m}^{2}\right)-4 q^{m}\left(a_{1 m} a_{2 m}+b_{1 m} b_{2 m}\right)}{1-q^{2 m}},
\end{aligned}
$$

the letters $\alpha, a, b$ representing vectors $\alpha_{i}, a_{1 i m}, b_{1 i m}, a_{2 i m}, b_{2 i m}(i=1,2, \cdots, n)$ defined by the following formulas:

† Loc. cit., formula (5.4). Correction: in the formula as there given the coefficient 4 should be deleted from the first term. This has as consequence the same correction in (5.6) and the first formula on page 359, also the removal of the term $3 \pi \alpha^{2}$ from (5.7), (5.8) and the second formula on page 357, none of which affects any result or proof of the cited paper. 


$$
\begin{aligned}
\alpha & =\frac{1}{2 \pi} \int_{0}^{2 \pi} g_{1}(\theta) d \theta-\frac{1}{2 \pi} \int_{0}^{2 \pi} g_{2}(\theta) d \theta, \\
a_{1 m} & =\frac{1}{\pi} \int_{0}^{2 \pi} g_{1}(\theta) \cos m \theta d \theta, \quad b_{1 m}=\frac{1}{\pi} \int_{0}^{2 \pi} g_{1}(\theta) \sin m \theta d \theta, \\
a_{2 m} & =\frac{1}{\pi} \int_{0}^{2 \pi} g_{2}(\theta) \cos m \theta d \theta, \quad b_{2 m}=\frac{1}{\pi} \int_{0}^{2 \pi} g_{2}(\theta) \sin m \theta d \theta .
\end{aligned}
$$

The connection between the present case of the one-sided surface and the two-contour case is expressed by the formulas

$$
\begin{gathered}
g_{1}(\theta)=g(\theta), g_{2}(\theta)=g(\theta+\pi), \\
A(g ; q)=\frac{1}{2} A\left(g_{1}, g_{2} ; q\right) .
\end{gathered}
$$

Hence, indicating the (vector) Fourier coefficients of $g(\theta)$ by $a_{m}, b_{m}$, we have evidently

$$
\begin{array}{ll}
a_{1 m}=a_{m}, & b_{1 m}=b_{m}, \\
a_{2 m}=(-1)^{m} a_{m}, & b_{2 m}=(-1)^{m} b_{m},
\end{array}
$$

so that by (6.1) we obtain

$$
\begin{aligned}
A(g ; q)= & \frac{\pi}{2} \sum_{m=1}^{\infty} m \frac{1-(-1)^{m} q^{m}}{1+(-1)^{m} q^{m}}\left(a_{m}{ }^{2}+b_{m}{ }^{2}\right) \\
= & \frac{\pi}{2}\left\{\frac{1+q}{1-q}\left(a_{1}{ }^{2}+b_{1}{ }^{2}\right)+2 \frac{1-q^{2}}{1+q^{2}}\left(a_{2}{ }^{2}+b_{2}{ }^{2}\right)\right. \\
& \left.+3 \frac{1+q^{3}}{1-q^{3}}\left(a_{3}{ }^{2}+b_{3}{ }^{2}\right)+4 \frac{1-q^{4}}{1+q^{4}}\left(a_{4}{ }^{2}+b_{4}{ }^{2}\right)+\cdots\right\} .
\end{aligned}
$$

To study $A(g ; q)$ in the neighborhood of $q=0$, we expand and rearrange this development according to powers of $q$, getting, after a little calculation,

$$
\begin{aligned}
A(g ; q)= & A(g)+\pi\left\{q\left(a_{1}{ }^{2}+b_{1}{ }^{2}\right)+q^{2}\left[\left(a_{1}{ }^{2}+b_{1}{ }^{2}\right)-2\left(a_{2}{ }^{2}+b_{2}{ }^{2}\right)\right]\right. \\
& +q^{3}\left[\left({a_{1}}^{2}+b_{1}{ }^{2}\right)+3\left(a_{3}{ }^{2}+b_{3}{ }^{2}\right)\right] \\
& +q^{4}\left[\left({a_{1}}^{2}+b_{1}{ }^{2}\right)+2\left(a_{2}{ }^{2}+b_{2}{ }^{2}\right)-4\left({a_{4}}^{2}+b_{4}{ }^{2}\right)\right] \\
& +q^{5}\left[\left({a_{1}}^{2}+b_{1}{ }^{2}\right)+5\left(a_{5}{ }^{2}+b_{5}{ }^{2}\right)\right] \\
& +q^{6}\left[\left({a_{1}}^{2}+b_{1}{ }^{2}\right)-2\left(a_{2}{ }^{2}+b_{2}{ }^{2}\right)+3\left(a_{3}{ }^{2}+b_{3}{ }^{2}\right)-6\left(a_{6}{ }^{2}+b_{6}{ }^{2}\right)\right] \\
& +q^{7}\left[\left({a_{1}}^{2}+b_{1}{ }^{2}\right)+7\left(a_{7}{ }^{2}+b_{7}{ }^{2}\right)\right] \\
& +q^{8}\left[\left(a_{1}{ }^{2}+b_{1}{ }^{2}\right)+2\left(a_{2}{ }^{2}+b_{2}{ }^{2}\right)+4\left({a_{4}}^{2}+b_{4}{ }^{2}\right)-8\left(a_{8}{ }^{2}+b_{8}{ }^{2}\right)\right] \\
& +\cdots\} .
\end{aligned}
$$


(It is to be recalled from One Contour that

$$
\left.A(g)=\frac{\pi}{2} \sum_{m=1}^{\infty} m\left(a_{m}{ }^{2}+b_{m}^{2}\right) .\right)
$$

The coefficients of the various powers of $q$ depend on the divisors of the exponent; they contain only positive terms if the exponent of $q$ is odd; while when this exponent is even, the term corresponding to any divisor of the exponent is + or - according as the codivisor is even or odd. These remarks describe completely the law according to which the series proceeds.

Let $g_{0}$ be the minimizing representation of $A(g)$ and $M$ the minimal surface determined by $g_{0}$, whose existence was proved in One Contour; $M$ is an ordinary two-sided surface, being a continuous image of the unit circular disc. The equations of $M$ are

$$
\begin{aligned}
x_{i} & =\Re F_{i}(w), \\
F_{i}(w) & =\sum_{m=0}^{\infty}\left(a_{i m}-i b_{i m}\right) w^{m}
\end{aligned}
$$

( $i$ the index running from 1 to $n$ not to be confused, of course, with $i$ the square root of -1 ), where $a_{i m}, b_{i m}$ are the Fourier coefficients of $g_{0 i}(\theta)$.

Suppose now that $M$ has a singular point (branch point) of the second order, corresponding to the center of the unit circle, $w=0$. This means, with the vectors $a_{m}, b_{m}$, that

$$
a_{1}^{2}+b_{1}^{2}=0, a_{2}^{2}+b_{2}^{2}>0 .
$$

Since $A\left(g_{0}\right)=m(\Gamma)$, we see that the expansion (6.6) then begins as follows:

$$
A\left(g_{0} ; q\right)=m(\Gamma)-2 \pi q^{2}\left(a_{2}{ }^{2}+b_{2}{ }^{2}\right)+\cdots .
$$

For sufficiently small $q$ these first two terms are dominant, and since the coefficient of $q^{2}$ is negative, we see that we have for all sufficiently small $q$

$$
A\left(g_{0} ; q\right)<m(\Gamma)
$$

thus $A(g ; q)$ takes values less than $m(\Gamma)$, consequently

$$
\bar{m}(\Gamma)=\min A(g ; q)<m(\Gamma),
$$

which is our fundamental sufficiency condition, ensuring therefore the existence of a one-sided minimal surface $\bar{M}$ bounded by $\Gamma$.

That the singular point was supposed to correspond to $w=0$ is evidently no restriction at all, since $g_{0}(\theta)$ is subject to arbitrary linear transformation $\dagger$

† One Contour, §6. 


$$
\tan \frac{\theta^{\prime}}{2}=\frac{a \tan \frac{\theta}{2}+b}{c \tan \frac{\theta}{2}+d}
$$

and two of the three arbitrary constants may be used to bring the singular point, wherever located in the interior of $M$, to correspond to $w=0$.

The same reasoning evidently extends to the case of a singular point of any even order $2 k$ :

$$
\begin{gathered}
a_{1}{ }^{2}+b_{1}{ }^{2}=0, a_{2}{ }^{2}+b_{2}{ }^{2}=0, \cdots, a_{2 k-1}{ }^{2}+b_{2 k-1}{ }^{2}=0, \\
a_{2 k}{ }^{2}+b_{2 k}{ }^{2}>0,
\end{gathered}
$$

the expansion of $A\left(g_{0} ; q\right)$ then beginning

$$
A\left(g_{0} ; q\right)=m(\Gamma)-2 k \pi q^{2 k}\left(a_{2 k}^{2}+b_{2 k}^{2}\right)+\cdots,
$$

so that again for all sufficiently small values of $q$ we have

$$
A\left(g_{0} ; q\right)<m(\Gamma),
$$

and therefore

$$
\bar{m}(\Gamma)<m(\Gamma) \text {. }
$$

In sum, we have (in the most important case of finite $m(\Gamma)$ ) the result stated in $\$ 2$ as Theorem II: if the minimal surface $M$ determined by $a$ contour $\Gamma$ has in its interior a singular point of even order, then a one-sided minimal surface $\bar{M}$ bounded by $\Gamma$ exists; the area of $\bar{M}$, moreover, is less than that of $M$.

Evidently this theorem applies to the contour in Fig. 1, where the surface $M$ has the general form of the Riemann surface for $z^{1 / 2}$, with a branch point of second order in its interior.

Examples in four-dimensional space are even easier to construct; for instance, the contour

$$
x_{1}=\cos 2 \theta, \quad x_{2}=\sin 2 \theta, \quad x_{3}=\cos 3 \theta, \quad x_{4}=\sin 3 \theta
$$

bounds the minimal surface

$$
\begin{aligned}
& x_{1}=\rho^{2} \cos 2 \theta, x_{2}=\rho^{2} \sin 2 \theta, \\
& x_{3}=\rho^{2} \cos 3 \theta, x_{4}=\rho^{2} \sin 3 \theta,
\end{aligned}
$$

on which the origin is a singular point of second order; the same contour is therefore the boundary of a one-sided minimal surface. 


\section{Arbitrary Jordan contour}

In all the preceding it has been assumed that $m(\Gamma)$ is finite (therefore also that $\bar{m}(\Gamma)$ is finite, by the inequality $\bar{m}(\Gamma) \leqq m(\Gamma))$. If the contour $\Gamma$ is sufficiently crinkly and tortuous, then $m(\Gamma)=+\infty$, or $A(g) \equiv+\infty$ (consequently $\bar{m}(\Gamma)=+\infty$, or $A(g ; q) \equiv+\infty$, by formulas $(2.14),(2.15))$, and it remains to consider the question of the existence of $\bar{M}$ for this case.

The discussion is not hard to carry through along the lines of the treatment of the similar question in Two Contours, $\$ 11$, and we give only the results.

Define

$$
e(\Gamma)=m(\Gamma)-\bar{m}(\Gamma) ;
$$

this definition is evidently significant so long as $m(\Gamma)$ is finite, and gives a value of $e(\Gamma) \geqq 0(<+\infty)$. We can then define, regardless of whether $m(\Gamma)$ is finite or $+\infty$ :

$$
\bar{e}(\Gamma)=\lim \sup e\left(\Gamma^{\prime}\right) \geqq 0(\leqq+\infty),
$$

where the variable contour $\Gamma^{\prime}$ approaches $\Gamma$ in every possible way subject only to the condition that $m\left(\Gamma^{\prime}\right)$ shall always be finite.

If now, with $m(\Gamma)=+\infty$, we have

$$
\bar{e}\left(\Gamma^{\prime}\right)>0
$$

then by regarding $\Gamma$ as limit of approaching contours $\Gamma^{\prime}$ with finite $m(\Gamma)$, it can be proved that a one-sided minimal surface $\bar{M}$ exists bounded by $\Gamma$; the area of $\bar{M}$ is $+\infty$.

If we shear off any strip, however narrow, all along the edge of $\bar{M}$, then the remaining part of $\bar{M}$ has finite area less than (or equal to) that of any other surface of the topology of a Möbius strip with the same boundary. $\dagger$ The infinite part of the area of $\bar{M}$ may thus be said to lie altogether on the edge of $\bar{M}$, which is perfectly regular in its interior but becomes infinitely curly towards its boundary.

Imagine, for example, a very thin tube about the curve of Fig. 1, and in this tube a highly crinkly and tortuous contour $\Gamma$, so that $m(\Gamma)=+\infty$, this contour following the general course of the original curve. Such a contour would be the boundary of a one-sided minimal surface of infinite area.

On the basis of the above considerations, the restriction of finite $m(\Gamma)$ can easily be removed from the singular point theorem of the preceding sec-

$\dagger$ Cf. J. Douglas, The least area property of the minimal surface determined by an arbitrary Jordan contour, Proceedings of the National Academy of Sciences, vol. 17 (1931), pp. 211-216. 
tion. If $m(\Gamma)=+\infty$, the surface $M$ still exists; suppose it has a branch point of even order $2 k$ corresponding to $w=0$.

In the formulas (2.14), (2.15) for $A(g ; q)$, let us separate the term $m=0$ from the others, writing

$$
\begin{aligned}
A(g ; q)= & \frac{1}{4 \pi} \int_{C} \int_{C} \sum_{i=1}^{n}\left[g_{i}(z)-g_{i}(\zeta)\right]^{2} \frac{d z d \zeta}{(z-\zeta)^{2}} \\
& +\frac{1}{4 \pi} \int_{C} \int_{C} \sum_{i=1}^{n}\left[g_{i}(z)-g_{i}(\zeta)\right]^{2} \sum_{m=-\infty}^{\infty} \frac{q^{m}}{\left[z-(-1)^{m} q^{m} \zeta\right]^{2}} d z d \zeta
\end{aligned}
$$

the accent attached to the last summation sign indicating the absence of $m=0$. The first term is $A(g)$, the second is a finite-valued continuous functional, if $\Gamma$ remains in any given finite region of space, on account of the uniform boundedness of the integrand; we denote this functional by $-B(g ; q)$, writing

$$
B(g ; q)=A(g)-A(g ; q) .
$$

Let $\Gamma^{\prime}$ be any contour with finite $m\left(\Gamma^{\prime}\right)$, and let $g_{0}^{\prime}$ be the representation of $\Gamma^{\prime}$ for which $m\left(\Gamma^{\prime}\right)$ is attained; then

$$
A\left(g_{0}^{\prime}\right)=m\left(\Gamma^{\prime}\right)
$$

while evidently

$$
A\left(g_{0}^{\prime} ; q\right) \geqq \bar{m}\left(\Gamma^{\prime}\right),
$$

hence, for all $q$,

$$
B\left(g_{0}^{\prime} ; q\right) \leqq m\left(\Gamma^{\prime}\right)-\bar{m}\left(\Gamma^{\prime}\right), \text { or } e\left(\Gamma^{\prime}\right) .
$$

Suppose now that $\Gamma$ is a contour with infinite $m(\Gamma)$, and let any sequence of contours $\Gamma^{\prime}$ with finite $m\left(\Gamma^{\prime}\right)$ approach $\Gamma$ as a limit so that $e\left(\Gamma^{\prime}\right)$ approaches a limit, $\lim e\left(\Gamma^{\prime}\right)$. By One Contour, $\$ 19$, a subsequence of these contours $\Gamma^{\prime}$ can be selected so that $g_{0}^{\prime}$ tends to $g_{0}$, the representation of $\Gamma$ which gives the minimal surface $M$. Let $\Gamma^{\prime}$ vary only in this subsequence; then, with $q$ any fixed value, (7.6) holds continually during the limit process, while

$$
\lim B\left(g_{0}^{\prime} ; q\right)=B\left(g_{0} ; q\right)
$$

by the continuity of the functional $B$. Consequently

$$
B\left(g_{0} ; q\right) \leqq \lim e\left(\Gamma^{\prime}\right),
$$

and a fortiori

$$
B\left(g_{0} ; q\right) \leqq \lim \sup e\left(\Gamma^{\prime}\right) \text {, or } \bar{e}(\Gamma)
$$


Now by the definition (7.5) and (6.6), we have the expansion of $B$ in powers of $q$ :

$$
\begin{aligned}
B\left(g_{0} ; q\right)= & \pi\left\{-q\left(a_{1}{ }^{2}+b_{1}{ }^{2}\right)\right. \\
& +q^{2}\left[-\left(a_{1}{ }^{2}+b_{1}{ }^{2}\right)+2\left(a_{2}{ }^{2}+b_{2}{ }^{2}\right)\right] \\
& -q^{3}\left[\left(a_{1}{ }^{2}+b_{1}{ }^{2}\right)+3\left(a_{3}{ }^{2}+b_{3}{ }^{2}\right)\right] \\
& +q^{4}\left[-\left(a_{1}{ }^{2}+b_{1}{ }^{2}\right)-2\left(a_{2}{ }^{2}+b_{2}{ }^{2}\right)+4\left(a_{4}{ }^{2}+b_{4}{ }^{2}\right)\right] \\
& +\cdots\},
\end{aligned}
$$

where $a, b$ are the Fourier coefficients of $g_{0}$, which figure also in the equations of $M$ :

$$
x_{i}=\Re F_{i}(w), F_{i}(w)=\sum_{m=0}^{\infty}\left(a_{i m}-i b_{i m}\right) w^{m} .
$$

Under the condition of a singular point of order $2 k$ on $M$ at $w=0$ :

$$
\begin{gathered}
a_{1}{ }^{2}+b_{1}{ }^{2}=0, a_{2}{ }^{2}+b_{2}{ }^{2}=0, \cdots, a_{2 k-1}{ }^{2}+b_{2 k-1}{ }^{2}=0, \\
a_{2 k}{ }^{2}+b_{2 k}{ }^{2}>0,
\end{gathered}
$$

the expansion (7.8) begins as follows:

$$
B\left(g_{0} ; q\right)=2 k \pi\left(a_{2 k^{2}}+b_{2 k}^{2}\right) q^{2 k}+\cdots .
$$

For sufficiently small $q$, the positive leading term gives its sign to the power series, so that for sufficiently small $q$

$$
B\left(g_{0} ; q\right)>0
$$

hence, by (7.7),

$$
\bar{e}(\Gamma)>0 \text {. }
$$

But this is the sufficient condition (7.3), which ensures the existence of a one-sided minimal surface $\bar{M}$ bounded by $\Gamma$.

Massachusetts Institute of Technology,

Cambridge, Mass. 